\title{
ESCISIÓN DE TORUS MANDIBULAR BILATERAL EN PACIENTE SISTÉMICAMENTE COMPROMETIDO: REPORTE DE CASO
}

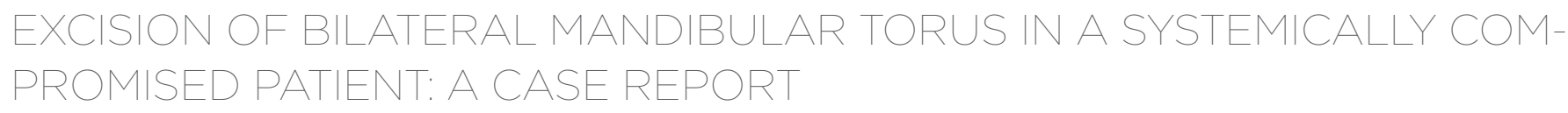

\section{Fabio Andrés Jiménez-Castellanos ${ }^{1 *}$ (1) fjimenezc@unicoc.edu.co}

\section{Dayana Andrea Arango-Piñeros ${ }^{1}$ (i)} daarango@unicoc.edu.co

\section{Camila Andrea Toloza-Cubillos ${ }^{1}$} camila.toloza29@gmail.com

Artículo recibido: 30/04/2020

Arbitrado por pares

Artículo aceptado: 22/10/2020

Artículo publicado: 10/12/2020

\section{Autor corresponsal:}

Fabio Andrés Jiménez-Castellanos fjimenezc@unicoc.edu.co
Citar como: Jiménez-Castellanos FA, Arango-Piñeros DA, Toloza-Cubillos CA. Escisión de torus mandibular bilateral en paciente sistémicamente comprometido: reporte de caso. Rev Cient Odontol (Lima). 2020; 8 (3): e035

DOI: $10.21142 / 2523-2754-0803-2020-035$

\section{RESUMEN}

Introducción: El torus mandibular es un crecimiento exofítico óseo ubicado en el área lingual de la mandíbula, generalmente bilateral. Puede estar asociado con trastornos temporomandibulares, afectar la pronunciación, producir halitosis, interferir con la deglución y causar dolor en la mucosa cuando está bajo prótesis mal diseñadas. Normalmente, el tratamiento no es necesario, excepto por necesidades protésicas, problemas funcionales, retención de alimentos, trastornos fonéticos o en caso de que se vaya a usar como injerto óseo autólogo. El objetivo de este reporte de caso fue realizar la escisión del torus mandibular bilateral en un paciente sistémicamente comprometido para su posterior rehabilitación con una prótesis parcial removible. Se realizo la atención de una paciente de 66 años sexo femenino, a quien, luego de analizar los medios diagnósticos y la evidencia científica, se le pudo realizar la escisión del torus mandibular bilateral de forma atraumática; esto permitió el correcto asentamiento de una prótesis parcial removible. La escisión del torus mandibular bilateral es un procedimiento seguro y predecible, y ayuda a evitar complicaciones protésicas, como el diseño de la estructura, y biológicas, como úlceras, depósitos de comida o halitosis, lo que devuelve la estabilidad y la función al sistema estomatognático

Palabras clave: exostosis, torus mandibular

\begin{abstract}
The mandibular torus is a bony exophytic growth located in the lingual area of the mandible. While this growth is generally bilateral, it can be associated with temporomandibular disorders. I It affects pronunciation, produces halitosis, interferes with swallowing, and causes pain in the mucosa under poorly designed prostheses. Normally, treatment is not necessary, except in cases with prosthetic needs, functional problems, food retention, phonetic disorders or if it is to be used as an autologous bone graft. The aim of this study was to perform a bilateral mandibular torus excision in a systemically compromised patient for subsequent rehabilitation with a removable partial denture. A 66-year-old female presenting a bilateral mandibular torus attended our dental clinic. After achieving the diagnosis and analyzing the scientific evidence available, the bilateral mandibular torus was atraumatically excised, allowing correct seating of a removable partial denture. Bilateral mandibular torus excision is a safe and effective procedure, which helps to avoid prosthetic complications involving structural design and biological complications such as ulcers, food deposits and / or halitosis, restoring stability and function to the stomatognathic system.
\end{abstract}

Keywords mesh: exostoses, mandibular torus

\footnotetext{
1 Facultad de Odontología, Institución Universitaria Colegios de Colombia (UNICOC). Bogotá, Colombia.
} 


\section{INTRODUCCIÓN}

Las exostosis son crecimientos benignos del tejido óseo, consideradas anomalías del desarrollo o variaciones anatómicas $\left({ }^{1}\right)$. Dos de las exostosis orales más comunes son el torus palatino (TP) y el torus mandibular (TM) ${ }^{(2)}$, que se componen principalmente de hueso cortical denso sin médula y están cubiertos por una mucosa delgada y poco vascularizada que tiende a crecer lenta y progresivamente $\left({ }^{3}\right)$. ElTP es una masa nodular exofítica de hueso que se eleva a lo largo de la sutura de la línea media del paladar duro. Por el contrario, el TM es un crecimiento exofítico óseo ubicado en el área lingual de la mandíbula en la región del canino o del premolar. Suelen ser, bilaterales y superiores a la línea milohioidea. Morfológicamente, varían en tamaño y se clasifican en planos, fusiformes, nodulares y lobulares $\left({ }^{2}\right)$.

La etiología de TM aún se desconoce, pero las causas postuladas incluyen factores genéticos, el trauma, los hábitos alimenticios y las afecciones sistémicas ( $\left.{ }^{4}\right)$. Morita et al. (2017) hallaron una prevalencia del TM del 58,3\% en 204 participantes, lo cual evidenció que estaba presente en más de la mitad de los participantes japoneses dentados sanos (quienes tenían un promedio de edad de 22 años) y se hallaba estrechamente asociado con el desgaste dental y el área de contacto oclusal $\left({ }^{5}\right)$. Sin embargo, existe gran variabilidad en diferentes grupos étnicos: $0,5 \%$ en indios brasileños, $8 \%$ en nigerianos, $31,9 \%$ en tailandeses, y se observa con mayor frecuencia en hombres que en mujeres $(6,7)$.

En la consulta, estos TM se hallan mediante el examen clínico intraoral de manera incidental, ya que normalmente no producen ningún síntoma. Sin embargo, pueden estar asociados con trastornos temporomandibulares, afectar la pronunciación, producir halitosis, interferir con la deglución y causar dolor en la mucosa bajo prótesis mal diseñadas $\left({ }^{8}\right)$. Generalmente, el tratamiento no es necesario, excepto por necesidades protésicas, problemas funcionales, retención de alimentos, trastornos fonéticos o en caso de que se vaya a usar como injerto óseo autólogo $\left({ }^{9}\right)$. Por lo tanto, el objetivo de este reporte de caso es realizar la escisión de torus mandibular bilateral en pacientes sistémicamente comprometidos, para su posterior rehabilitación con una prótesis parcial removible. Este reporte de caso se describe según la guía CARE.

\section{REPORTE DE CASO}

Paciente de 66 años, de sexo femenino, con las siguientes afecciones sistémicas controladas: hipotiroidismo, síndrome de la silla turca vacía, apnea del sueño e hipertensión, llega a consulta al posgrado de periodoncia en la Institución Universitaria Colegios de Colombia (UNICOC), en mayo de 2017. Fue remitida del posgrado de rehabilitación oral, para la extirpación de TM bilateral, puesto que requería la restauración del sector inferior posterior, por medio de una prótesis parcial removible. Se realizó un análisis extraoral e intraoral, se evaluaron las imágenes diagnósticas y una historia clínica minuciosa, se realizaron exámenes de laboratorio y se verificó que estuviera libre de focos infecciosos, como enfermedad periodontal activa y lesiones cariosas. Se tomaron impresiones y se evaluaron tanto los modelos de estudio en articulador Whip Mix 2240 como las fotos intraorales (figura 1).

Por lo tanto, se determinó la necesidad de extirpar el TM bilateral para favorecer el asentamiento y la adaptación de la prótesis parcial removible inferior. Se explicaron las ventajas y desventajas del tratamiento, con los posibles riesgos y complicaciones. $\mathrm{La}$

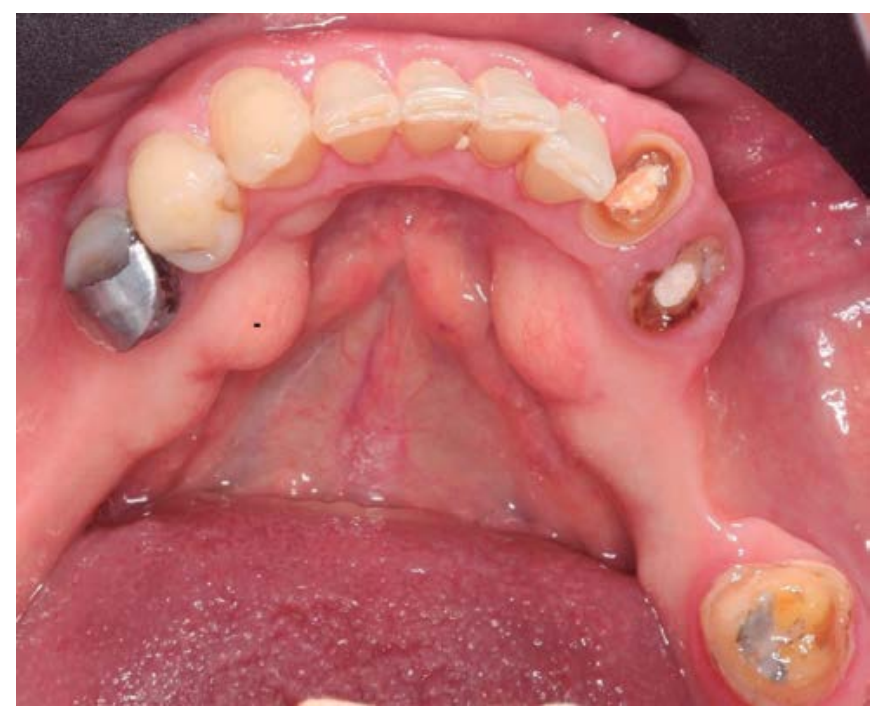

Fig. I. Análisis intraoral inicial de la zona 
paciente aceptó el tratamiento sugerido y firmó un consentimiento informado. Se realiza el procedimiento quirúrgico, asepsia y antisepsia, mediante la aplicación de iodopovidona al $8 \%$ extraoral y un enjuague con clorhexidina al $2 \%$ por 30 segundos. Se aplicó anestesia infiltrativa con lidocaina al $2 \%$, con epinefrina 1:80000, previa aspiración en la zona vestibular y lingual de todo el maxilar inferior. Luego incisión con hoja de bisturí n. ${ }^{\circ}$ $15 \mathrm{c}$ intrasulcular del 48, paracrestal hacia vestibular en zona de torus bilaterales, y se continuó con incisiones intrasulculares en área lingual del 35 al 43. Se elevó el colgajo a espesor total con periostotomo de Buser, se posicionó una fresa cilíndrica diamantada de $1 \mathrm{~mm}$ de diámetro entre el TM y el reborde alveolar, y se seccionó hasta dejar solamente $2 \mathrm{~mm}$ de unión apical entre el TM y el reborde alveolar, y se terminó de retirar con un cincel quirúrgico. Posteriormente, se realizó el biselado de espículas óseas con la fresa diamantada, se reposicionó el colgajo y se suturó con ácido poliglicólico 4/0, con una aguja cortante (figuras 2 y 3). Finalmente, se envíó a patología oral con un diagnóstico presuntivo de torus

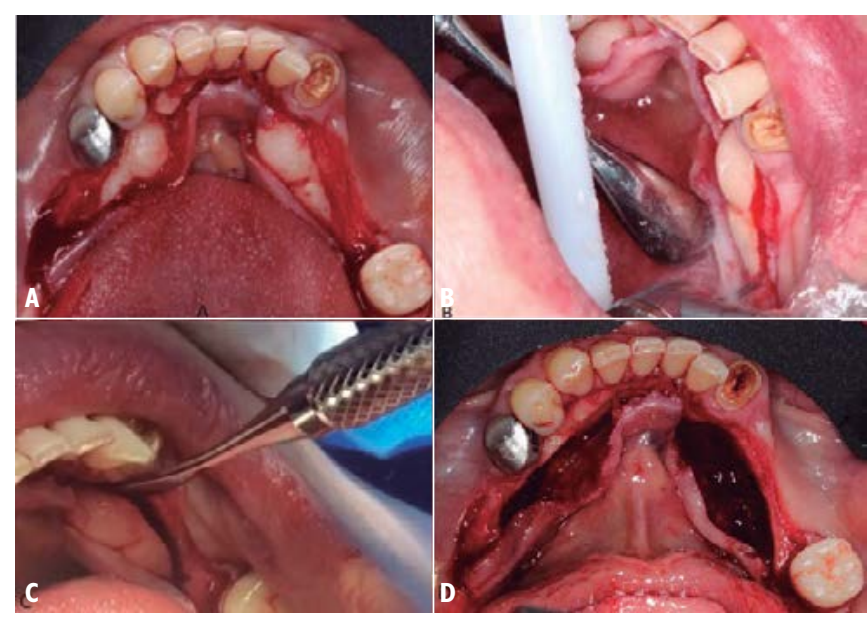

Fig. 2. A. Incisiones, colgajo. B y C. Delimitación torus mandibular. D. Extirpación del torus mandibular

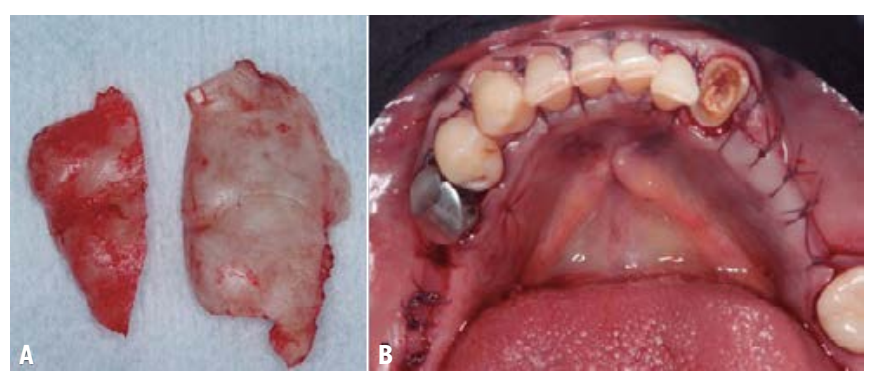

Fig. 3. A. Torus Mandibular. B. Sutura mandibular, resultado que se confirmó luego del análisis del TM. La paciente no presentó complicaciones intra ni posoperatorias. Se recetó acetaminofén (tabletas de $500 \mathrm{mg}, 1$ tableta cada 6 horas por 3 días) y amoxicilina (cápsulas de $500 \mathrm{mg}, 1$ cápsula cada 8 horas por 7 días).

A los 8 días, se retiraron las suturas y se realizó un control adicional a los 15 días. Luego, comenzó todo el proceso de rehabilitación oral integral. La paciente regresa luego de un año de haber realizado la extirpación del torus mandibular, por motivos personales; en ese momento se tomó la impresión definitiva. Veinte días después se realizó la entrega de la prótesis parcial removible inferior, en la que el conector mayor fue una barra lingual. La paciente aún continúa con controles semestrales (figura4).

La paciente está conforme y satisfecha con el tratamiento realizado.

\section{DISCUSIÓN}

Para lograr una escisión exitosa del TM, es necesario conocer su histología, la cual demuestra una organización similar al hueso normal. Regularmente, el TM está formado por hueso cortical con la presencia central de tejido óseo esponjoso, canales de Havers, osteocitos normales y áreas dispersas de tejido conectivo que contienen vasos sanguíneos dilatados $\left({ }^{10}\right)$. Sin embargo, Kün-Darbois et al. (2017) informaron la presencia de

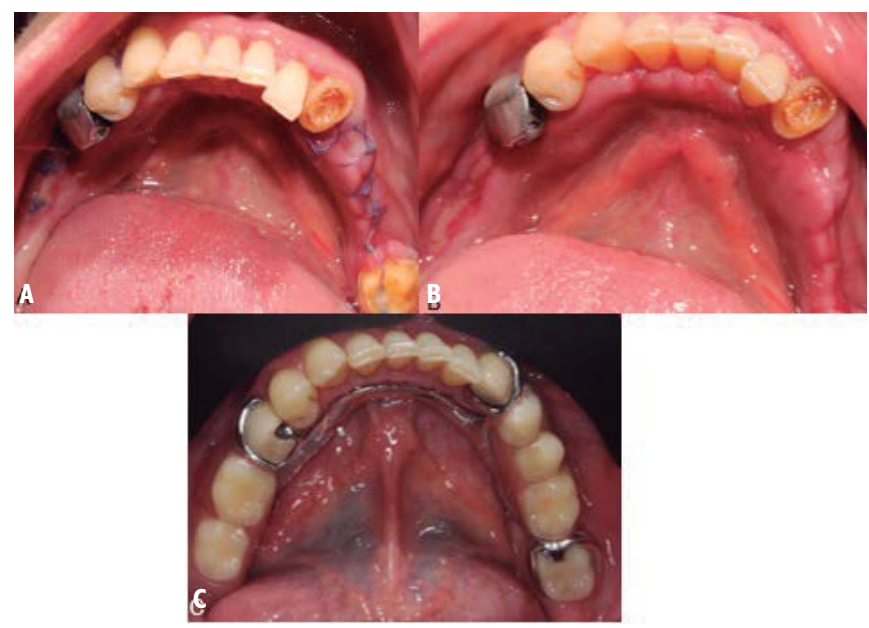

Fig. 4. A. Cicatrización 8 días. B. Cicatrización 15 días. C. Entrega de rehabilitación definitiva 12 meses luego del procedimiento quirúrgico 
numerosos canales haversianos aleatorios, en contraste con la orientación paralela en un reborde mandibular normal, y mayor presencia de osteoclastos que generan una remodelación ósea activa y asimétrica $\left({ }^{11}\right)$.

La presencia de TM promueve cambios morfológicos y funcionales en el sistema estomatognático, como lo demostraron Mendes da Silva et al. (2017) quienes utilizaron la electromiografía para analizar el rendimiento de los músculos masticatorios en presencia de TM, encontrando hiperactividad de estos, mayor fuerza de mordida y volumen muscular reducido en los músculos temporales $\left({ }^{12}\right)$. Por otro lado, la paciente en la anamnesis reportó diagnóstico de apnea del sueño, un trastorno que causa hipoxia intermitente debido a un colapso parcial o completo de las vías respiratorias durante el sueño. Existen varias enfermedades relacionadas con la apnea del sueño, incluida la enfermedad cardiovascular, las enfermedades endocrinas, la enfermedad cerebrovascular y el trastorno cognitivo. Por lo tanto, el diagnóstico temprano de la apnea del sueño y el tratamiento adecuado son muy importantes para minimizar el riesgo de enfermedades comórbidas $\left({ }^{13}\right)$.

Recientemente, Ahn et al. (2019) concluyeron que la presencia de TM aumenta la severidad de la apnea del sueño y que se requerían más estudios clínicos donde se evalúe las consecuencias positivas y negativas de la escisión del TM $\left.{ }^{(14}\right)$. En concordancia con nuestro reporte de caso, la paciente reporta mejoría un año luego de la escisión del TM en cuanto al diagnóstico de apnea del sueño que presenta. El TM se ha reportado también como una zona donadora de injerto óseo autógeno para los procedimientos de regeneración ósea guiada horizontal y vertical, la elevación de seno maxilar y la regeneración tisular de defectos óseos periodontales $\left({ }^{15-17}\right)$. Sin embargo, en nuestro caso, a pesar de que se le ofreció a la paciente que podía optar por una prótesis implantosoportada, informó que no tenía los recursos económicos ni la disposición para acceder a ese tipo de tratamiento, por lo que se decidió por una prótesis parcial removible y por ello resultó necesario la escisión del TM. Este procedimiento, normalmente, resulta seguro y predecible con secuelas posoperatorias mínimas. Hay que tener precaución en el momento de realizar el diseño del colgajo debido a que la mucosa que lo rodea es muy delgada y avascular. Se han utilizado diversos instrumentos tanto como para la incisión (bisturí, electrocirugía, láser) como para la escisión del TM (pieza de baja velocidad, pieza de alta velocidad o en combinación con cinceles quirúrgicos, láser) $\left({ }^{18,19}\right)$. Sin embargo, no hay ningún estudio que compare la morbilidad de las distintas técnicas.

Entre las complicaciones más comunes están las siguientes: dolor posoperatorio, edema, espículas óseas, lesiones del conducto salival, lesión del nervio lingual, perforación de la tabla ósea lingual, enfisema, dehiscencia de la herida e infección $\left({ }^{19}\right)$. Para minimizar estas complicaciones, se optó por realizar una técnica quirúrgica lo más conservadora posible, usando fresas diamantadas de grano fino y solo cuando quedaban 2 mm de unión con el reborde se utilizó el cincel quirúrgico para disminuir los golpes en el paciente, que suelen ser molestos e incómodos. Por último, una complicación menos común es la recurrencia del TM. Brunsvold et al. (1995) reportaron 2 casos con un periodo de seguimiento de 11 y 14 años, y observaron una recurrencia de alrededor del 60\% del TM, aunque no es clara la causa por la cual se puede presentar este tipo de manifestación clínica, pero es necesario informar la posible recidiva al paciente antes de la escisión quirúrgica $\left({ }^{20}\right)$.

\section{CONCLUSIONES}

La escisión del TM bilateral es un procedimiento seguro y predecible, que ayuda a evitar complicaciones protésicas, como el diseño de la estructura, y biológicas, como úlceras, depósitos de comida o halitosis. De esta manera, devuelve la estabilidad y la función al sistema estomatognático. Adicionalmente, no se observó recurrencia del TM bilateral en el periodo de seguimiento de 12 meses. Sin embargo, debe mantenerse en control debido a que en la literatura se observa recurrencia en periodos de seguimiento más largos, de hasta 11 años. 
Contribución de los autores: Fabio Andrés Jiménez Castellanos contribuyó en la cirugía y la redacción del reporte de caso. Dayana Andrea Arango Piñeros contribuyó en la cirugía, los controles posquirúrgicos y la revisión de literatura. Camila Andrea Toloza Cubillos contribuyó en la rehabilitación oral, la fotografía y la revisión de literatura.

Fuentes de Financiamiento: Autofinanciada.

Potenciales conflictos de interés: Ninguno.

\section{REFERENCIAS BIBLIOGRÁFICAS}

1. Koç N, Çağırankaya LB. Mandibular tori are associated with mandibular bone quality: a case-control study. Folia Morphol (Warsz). 2018; 77 (4): 736-41. doi: 10.5603/FM.a2018.0094

2. Antoniades DZ, Belazi M, Papanayiotou P. Concurrence of torus palatinus with palatal and buccal exostoses: case report and review of the literature. Oral Surg Oral Med Oral Pathol Oral Radiol Endod. 1998; 85 (5): 552-7. doi: 10.1016/s10792104(98)90290-6

3. García-García AS, Martínez-González JM, Gómez-Font R, Soto-Rivadeneira A, Oviedo-Roldán L. Current status of the torus palatinus and torus mandibularis. Med Oral Patol Oral Cir Bucal. 2010; 15 (2): 353-60. doi: 10.4317/medoral.15.e353

4. Morrison MD, Tamimi F. Oral tori are associated with local mechanical and systemic factors: a case-control study. J Oral Maxillofac Surg. 2013; 71 (1): 14-22. doi: 10.1016/j. joms.2012.08.005

5. Morita K, Tsuka H, Shintani T, Yoshida M, Kurihara H, Tsuga K. Prevalence of Torus mandibularis in young healthy dentate adults. J Oral Maxillofac Surg. 2017; 75 (12): 2593-8. doi: 10.1016/j.joms.2017.04.044

6. Bernaba JM. Morphology and incidence of torus palantinus and mandibularis in Brazilian indians. J Dent Res. 1977; 56 (5): 499501. doi: 10.1177/00220345770560050901

7. Maduakor SN, Nwoga MC. Prevalence of mandibular and palatine tori among the Ibos in Enugu, South-East Nigeria. Niger J Clin Pract. 2017; 20 (1): 57-60. doi: 10.4103/11193077.178911

8. Bertazzo-Silveira E, Stuginski-Barbosa J, Porporatti AL, Dick B, Flores-Mir C, Manfredini D, et al. Association between signs and symptoms of bruxism and presence of tori: a systematic review. Clin Oral Investig. 2017; 21 (9): 2789-99. doi: 10.1007/ s00784-017-2081-7

9. Dou XW, Park W, Lee S, Zhang QZ, Carrasco LR, Le AD. Loss of notch3 signaling enhances osteogenesis of mesenchymal stem cells from mandibular torus. J Dent Res. 2017; 96 (3): 347-54. doi:10.1177/0022034516680349

10. Ephros H, Klein R, Sallustio A. Preprosthetic Surgery. Oral Maxillofac Surg Clin North Am. 2015; 27 (3): 459-72. doi: 10.1016/j.coms.2015.04.002
11. Kün-Darbois JD, Guillaume B, Chappard D. Asymmetric bone remodeling in mandibular and maxillary tori. Clin Oral Investig. 2017; 21 (9): 2781-8. doi: 10.1007/s00784-017-2080-8

12. Mendes da Silva J, Pérola Dos Anjos Braga Pires C, Mendes Rodrigues LA, Palinkas M, De Luca Canto G, Batista de Vasconcelos P, et al. Influence of mandibular tori on stomatognathic system function. Cranio. 2017; 35 (1): 30-7. doi: 10.1080/08869634.2015.1122417

13. Buratti L, Viticchi G, Baldinelli S, Falsetti L, Luzzi S, Pulcini A, et al. Sleep apnea, cognitive profile, and vascular changes: an intriguing relationship. J Alzheimers Dis. 2017; 60 (3): 11951203. doi: 10.3233/JAD-170445

14. Ahn SH, Ha JG, Kim JW, Lee YW, Yoon JH, Kim CH, et al. Torus mandibularis affects the severity and position-dependent sleep apnoea in non-obese patients. Clin Otolaryngol. 2019; 44 (3): 279-85. doi: 10.1111/coa.13286

15. Jeff Wang CW, Bruce Huang CH, Grossman SH, Pourati J. Vertical ridge augmentation with mandibular lingual torus block graft. J Oral Implantol. 2016; 42 (4): 369-72. doi: 10.1563/aaidjoi-D-15-00151

16. Karaca IR, Ozturk DN, Akinci HO. Mandibular torus harvesting for sinus augmentation: two-year follow-up. J Maxillofac Oral Surg. 2019; 18 (1): 61-4. doi: 10.1007/s12663-018-1135-y

17. Hassan KS, Al-Agal A, Abdel-Hady AI, Swelam WM, Elgazzar RF. Mandibular tori as bone grafts: an alternative treatment for periodontal osseous defects - clinical, radiographic and histologic morphology evaluation. J Contemp Dent Pract. 2015; 16 (3): 192-200. doi: 10.5005/jp-journals-10024-1660

18. Gounder R, Gounder S. Laser science and its applications in prosthetic rehabilitation. J Lasers Med Sci. 2016; 7 (4): 209-13. doi: 10.15171/jlms.2016.37

19. Martínez G, Cabrera G, Surgical removal of mandibular torus: case report. Revista Oral. 2016; 17 (53): 1324-7.

20. Brunsvold MA, Kaiser DA, Faner RM. Recurrence of mandibular tori after surgical removal: two case reports. J Prosthodont. 1995; 4 (3): 164-7. doi: 10.1111/j.1532-849x.1995.tb00334.x 